\title{
The combination of Raman spectroscopy and Autofluorescence analysis for estimation of blood and urine homeostasis
}

\author{
L.A. Shamina ${ }^{1}$, I.A. Bratchenko ${ }^{1}$ \\ ${ }^{1}$ Samara National Research University, 34 Moskovskoe Shosse, 443086, Samara, Russia
}

\begin{abstract}
In this study we measured spectral features of blood and urine by Raman spectroscopy and autofluorescence analysis. Analysis of specific spectra allows for identification of informative spectral bands proportional to components whose content is associated with body fluids homeostasis changes at various pathological conditions. In general, the developed approach of body fluids analysis provides the basis of a useful and minimally invasive method of pathologies screening.
\end{abstract}

Keywords: Raman spectroscopy; autofluorescence; biofluidity homeostasis; blood; urine; pathology

\section{Introduction}

The pathological conditions provoke alterations in body fluids homeostasis; therefore it is possible to use the component composition analysis of urine, blood, saliva and other body fluids for pathologies detection such as cancer [1]. Presently, the biochemical analyses are widely used for the body fluids cancer diagnosis. In addition to the laboratory methods today a variety of physical and chemical methods of analysis may be successfully utilized for the study of the body fluids composition. Physical methods have such advantages as simplicity of sample preparation, wide dynamic range and great versatility in comparison with chemical methods of analysis. Therefore, body fluids analysis with optical methods can become a successful alternative to existing laboratory methods. Raman Spectroscopy (RS) and autofluorescence (AF) analysis allow for the homeostasis changes detection in the body fluids at the molecular level [2]. These techniques are successfully used in different branches of clinical medicine and in the experimental studies of the body fluids composition for the various locations cancer detection. The aim of this work is to study the spectral features of blood and urine from patients with tumors for identification criteria that may allow to estimate the homeostasis changes and the tumor presence.

\section{Materials and methods}

\subsection{Experimental setup}

Study of the body fluids spectral features was performed with the experimental setup shown in Fig. 1. The excitation of collected spectra was performed by the laser module LuxxMaster LML-785.0RB-04 (central wavelength $785 \mathrm{~nm}$ ). The fiberoptic Raman probe RPB785 allows for focusing of the exiting radiation, collecting and filtering of the scattered radiation. The collected signal was decomposed into a spectrum using a high-resolution Shamrock SR-500i-D1-R spectrograph with integrated cooled up to $-65^{\circ} \mathrm{C}$ digital camera ANDOR DU416A-LDC-DD. Tested body fluids were placed in the PMMA cuvette with an aluminum coating. The cuvette geometry (depth $6.5 \mathrm{~mm}$, radius of deepening curvature $19 \mathrm{~mm}$ ) was optimized to match the working distance of probe focusing lens. The Raman probe was normally positioned on the axis of the deepening; a detailed description of the utilized experimental setup is presented in [3]. The utilized spectrograph with a grating of $600 \mathrm{slits} / \mathrm{mm}$ allows for recording the spectrum of the tested substance in 780-950 $\mathrm{nm}$ area divided by three spectral ranges; for each single spectral range the exposure time was 20 seconds. A sequential recording of three spectra for each tested sample was performed. The final spectrum was received from averaging of all three recorded spectra. The total time of the final spectrum recording was 3 minutes.

\subsection{Samples preparation}

The standardized collection of blood and urine samples from patients of Samara Regional Clinical Oncology Dispensary was performed. Collected samples were placed in sterile test-tubes and were stored at $+2+4{ }^{\circ} \mathrm{C}$ before the analysis. Analysis of collected body fluids was performed within $60 \mathrm{~h}$ after sample collection. Patients of Samara Regional Clinical Oncology Dispensary with malignant tumors or benign tumors were enrolled in this study. Patients with systemic diseases and patients taking any medical antitumor drugs were excluded from the study.

\subsection{Spectra processing}

Recorded spectra were processed by the method proposed by Zeng et al [4] for AF and Raman signals separation. The processing of experimental data was performed on the bases of regression analysis. Definition of spectrum informative bands during the regression model constructing was performed by the analysis of the variable importance in projection (VIP) [5]. The 
VIP distribution makes it possible to define the most informative spectral bands in the blood and urine spectra specific for patients with lung cancer.

\section{Results}

\subsection{Spectral characteristics of blood}

Currently a set of biochemical methods for analyzing body fluids aimed to detect a pathological process is used in laboratory diagnostics. Biochemical methods make it possible to perform a quantitative estimation of certain organic and mineral components level, as well as that of enzymes and hormones and to detect their deviations from the norm [6]. Qualitative estimation of the level of indicators included in the standard biochemical analysis is possible when studying the spectral characteristics of body fluids. Figure 1 shows the common Raman spectrum of blood.

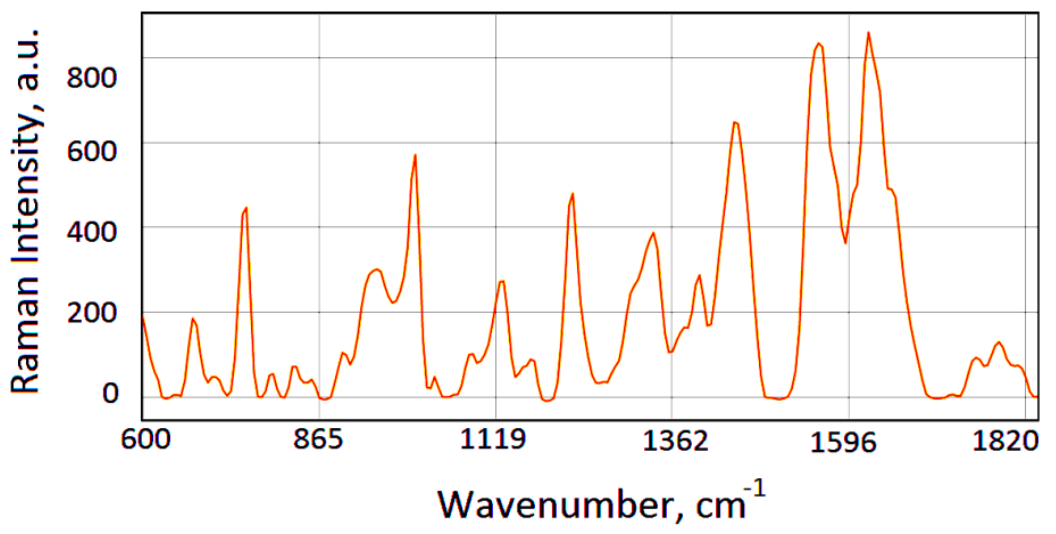

Fig.1. Common Raman spectrum of blood.

Human body fluids have a complex chemical composition; shape of body fluids Raman spectra and certain spectral bands intensities are due to the contribution of molecular vibrations of several components. Therefore, the analysis of the intensity ratios of certain bands of the body fluid spectrum allows to assess homeostasis changes and to obtain the information about the functional state and possible pathologies of internal organs. The first step in our study is comparison of the obtained body fluid spectral characteristics with the spectral bands associated with components analyzed in standard biochemical analysis. Table 1 shows the observed vibrational bands of human blood Raman spectra. Here general organic components of biochemical blood test are marked with $(*)$ symbol; experimental peaks observed in our study are in bold type.

Table 1. Observed vibrational bands of human blood Raman spectra. Here general organic components of biochemical blood test are marked with (*) symbol; experimental peaks observed in our study are in bold type.

\begin{tabular}{|c|c|c|}
\hline Wavenumber, $\mathrm{cm}^{-1}$ & Substances & References \\
\hline 493 & Bilirubin $^{*}$ & [7] \\
\hline 679 & Creatinine $^{*}$ & [8] \\
\hline 756 & L-tryptophan (is part of several organic & [9] \\
\hline & components) & \\
\hline 829 & Collagen & {$[10]$} \\
\hline 846 & Creatinine $^{*}$ & {$[8]$} \\
\hline 941 & Protein* & {$[11]$} \\
\hline \multirow[t]{3}{*}{1002} & Protein* & [11] \\
\hline & Urea* & {$[8]$} \\
\hline & Hemoglobin* & [12] \\
\hline 1128 & Glucose $^{*}$ & [13] \\
\hline 1225 & L-tryptophan (is part of several organic & [9] \\
\hline \multirow{3}{*}{1336} & components) & \\
\hline & Protein* & [11] \\
\hline & Bilirubin* & [7] \\
\hline \multirow[t]{2}{*}{1451} & Protein ${ }^{*}$ & [11] \\
\hline & Bilirubin* $^{*}$ & [7] \\
\hline 1500 & Bilirubin $^{*}$ & [7] \\
\hline \multirow[t]{2}{*}{1556} & Hemoglobin* & [12] \\
\hline & Fibrin & [12] \\
\hline 1623 & Hemoglobin* & [12] \\
\hline
\end{tabular}

Analysis of Table 1 allows for defining that the blood spectral characteristics recorded by the utilized experimental setup contain intensity peaks proportional to the main organic components determined by biochemical analysis. However, the homeostasis state estimation only by certain peaks provides insufficient information for the detection of the pathologies such as cancer, since the presented corresponding components have low specificity for certain cancer localization detection. In addition, the tumor cells metabolism and its interaction with the microenvironment are complex; tumor-associated metabolic changes may significantly differ for various tumors [14]. It should be stressed that human body fluids have a complex chemical composition, and the blood spectral characteristics are due to the presence of a large number components including ones with partially 
overlapping spectra. Particularly, as shown in Table 1, the spectral characteristics of proteins, urea and hemoglobin simultaneously contribute to the band $1002 \mathrm{~cm}-1$; the intensities of the bands $1336 \mathrm{~cm}-1$ and $1451 \mathrm{~cm}-1$ are proportional to the changes of proteins and bilirubin; hemoglobin and fibrin contribute to the band $1556 \mathrm{~cm}-1$. Moreover, the intensity of the bands $756 \mathrm{~cm}-1$ and $1225 \mathrm{~cm}-1$ is proportional to the concentration of L-tryptophan, which is part of several organic components. Thus, the detection of the position and intensity of Raman peaks may not be sufficient for detection of pathology by body fluid spectral analysis. A more detailed analysis of the body fluid component composition is required for pathology identification; therefore, it is necessary to detect and evaluate the spectral properties of those chemical components that are characteristic of a particular pathology. In this case, obtaining statistically reliable information is possible by using multidimensional processing of the full body fluid spectrum. For this purpose, the experimental data were processed on the bases of discriminant analysis method with regression on latent structures (PLS-DA). VIP allows for evaluation of individual variables from the predictors block influence on the PLS model. The higher the VIP-score of an individual variable is, the more significant it is in model construction. VIP-scores of Raman spectra matrix of blood samples are shown in Fig. 2.

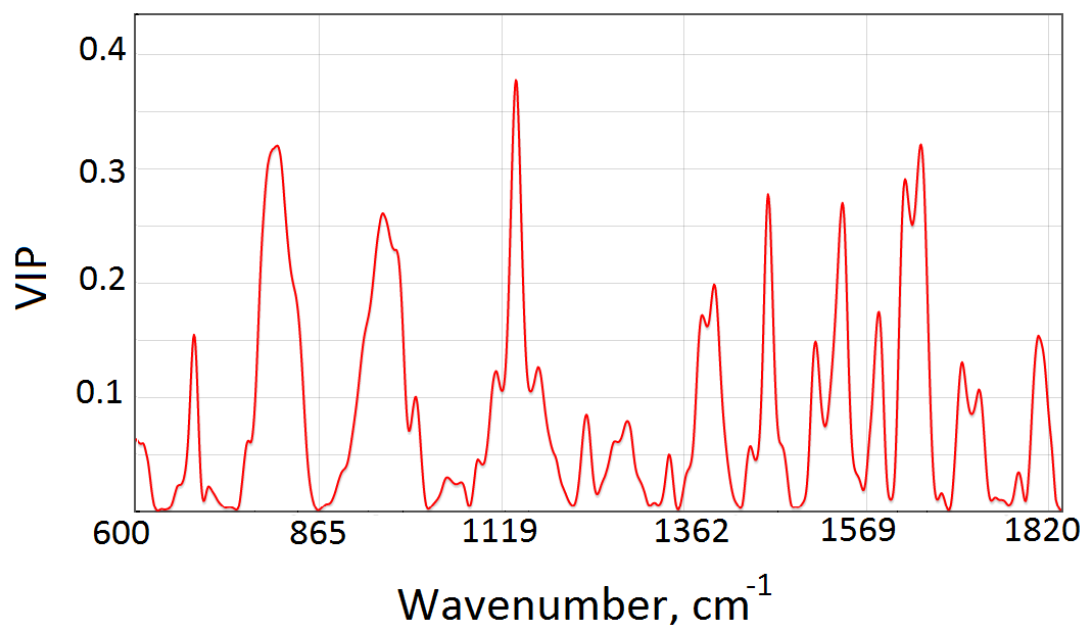

Fig. 2. VIP-scores of blood samples Raman spectra matrix.

Analysis of Fig. 2 allows for defining the most informative spectral bands for identification the features of blood Raman spectra in case lung cancer growth. The multivariate analysis of the experimental data allows to establish that the informative criteria for the lung cancer detection are changes in the Raman bands intensity 790-820 cm-1 (glutathione), 1135-1140 cm-1 (mannose), 946-970 cm-1 (proteins), 1465-1475 cm-1 (lipids, proteins) and 1640-1660 cm-1 (proteins, phospholipids) [3].

\subsection{Spectral characteristics of urine}

Approximation curves of urine AF for tested samples are shown in Fig. 3.

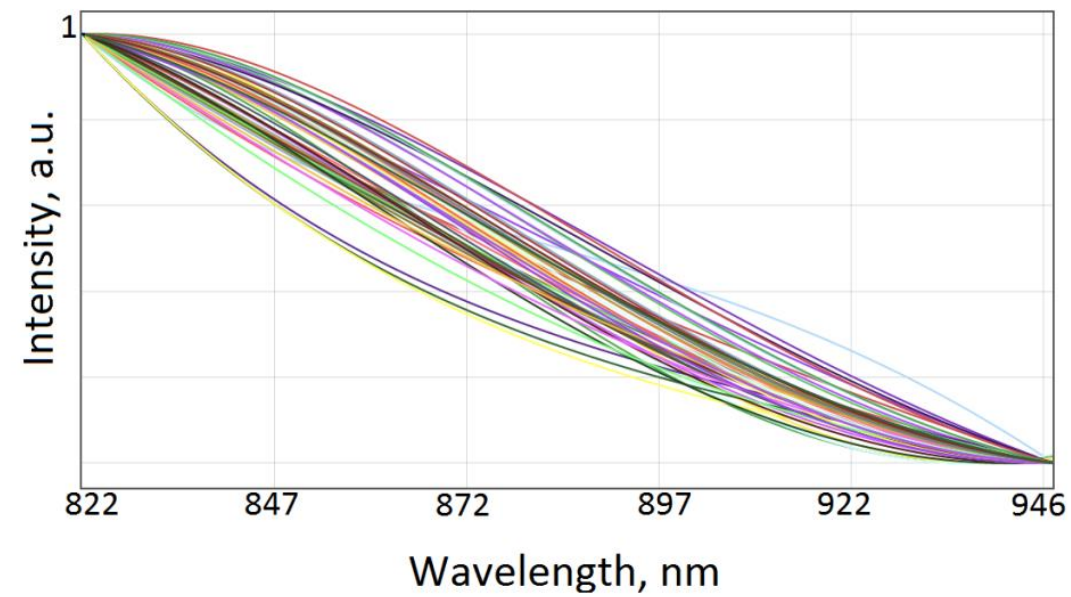

Fig. 3. Polynomial approximation of urine samples AF.

The porphyrins (nitrogen-containing pigments) accumulates in sites of active cells division and excretes with urine. Alterations in the AF urine spectrum reflect changes and metabolic imbalance of porphyrins. Therefore, the AF intensity of urine can be used as an informative criterion of oncopathology growth. The AF spectrum of porphyrins has features in red and near-infrared spectral ranges, so the excitation of the AF spectra by $785 \mathrm{~nm}$ laser allows to evaluate the presence of porphyrins in the tested sample. Analysis of the VIP-scores of urine samples Raman spectra matrix allows to determinate that for oncopathology the most specific changes in urine homeostasis are associated with the spectral bands 1000-1015 cm-1 (urea), 
1525-1560 cm-1 (tryptophan, proteins), and 1690-1705 cm-1 (pyruvate). An example use of a RS and AF combination for the analysis of body fluids in the detection of oncopathology was demonstrated in [3].

\section{Discussion and conclusions}

Detected AF and RS spectra features may be the basis of the method for pathologies detection and become an alternative to available detection techniques of pathological conditions using laboratory methods for body fluids analysis. Besides, in future studies it is possible to analyze the correlation of the body fluids spectral characteristics and the biochemical studies results, which may allow to expand the description of the various components contribution to the observed spectral bands. In addition to studied spectral properties of urine and blood, it is also possible to use additional body fluids as research objects for noninvasive diagnostics of various pathologies. The developed approach may become the basis for the non-invasive method of cancer screening, for example, when used in Lab-on-a-chip (LOC) systems [15]. The advantages of such systems are portability, small amount of the tested sample and high efficiency. Utilizing the LOC system may simplify the current experimental setup and replace an expensive spectrometer and a cooled digital camera with a less costly portable spectrometer. Improving the recorded signal quality is also possible due to the utilizing higher quality optical elements (filters), as we used low cost Raman Probe in this research. Moreover, it is possible to increase the accuracy of detection of particular pathology by preallotment of certain markers from tested samples. This approach was demonstrated by Feng et al [16]. For this purpose it is expedient to use, for example, microfluidics technologies and chromatography. Utilizing such technologies allows for sequential chemical selection of body fluids components including the stages of sample separation into different fractions, mixing the intermediate products and their transfer to various reaction microchambers. However, in case of body fluids microdose spectra collection the recorded signal quality may decrease. Improvement of the Raman signal collection is possible with the application of Surfaceenhanced Raman spectroscopy (SERS). SERS allows to achieve an improvement of registered Raman signal by several orders and is successfully used in the analysis of various biological material microdoses. Thus, the LOC system, including microfluidics and SERS technologies will improve the obtained spectra quality and increase the informativeness of the analysis. In general, the development of LOC system based on proposed method may provide the opportunity of human body fluids precise analysis for accurate screening of pathologies and detection of microorganisms in body fluids.

\section{Acknowledgments}

This research was supported by the Ministry of Education and Science of the Russian Federation and the program U.M.N.I.K.

\section{References}

[1] Peedell C. Concise Clinical Oncology. Elsevier Health Sciences, 2005; 395 p.

[2] Tuchin V. Handbook of Optical Biomedical Diagnostics. SPIE Press Book, 2002; 1410 p.

[3] Shamina L, Bratchenko IA, Artemyev DN, Myakinin OO, Moryatov AA, Kaganov OI, Orlov AE, Kozlov SV, Zakharov VP. Raman and autofluorescence analysis of human body fluids from patients with malignant tumors. J .of Biomedical Photonics \& Eng . 2017; 3(2).

[4] Zeng H. Automated autofluorescence background subtraction algorithm for biomedical Raman spectroscopy. Applied Spectroscopy 2007; 61(11): 12251232.

[5] Farrés M, Platikanov S, Tsakovski S, Tauler R. Comparison of the variable importance in projection (VIP) and of the selectivity ratio (SR) methods for variable selection and interpretation. Journal of Chemometrics 2015; 29(15): 528-536.

[6] Glick D. Methods of Biochemical Analysis. John Wiley \& Sons, 2009; 540 p.

[7] Celis F, Campos-Vallette MM, Gómez-Jeria JS, Clavijo RE, Jara GP, Garrido C. Surface-enhanced Raman scattering and theoretical study of the bilichromes biliverdin and bilirubin. Spectroscopy Letters 2016; 49(5): 336-342.

[8] de Almeida ML, Saatkamp CJ, Fernandes AB, Pinheiro AL, Silveira LJr. Estimating the concentration of urea and creatinine in the human serum of normal and dialysis patients through Raman spectroscopy. Lasers Med Sci. 2016; 31(7): 1415-1423.

[9] Gelder J, de Gussem K, Vandenabeele P, Moens L. Reference database of Raman spectra of biological molecules. J. Raman Spectrosc 2007; 38(9): 11331147.

[10] Lin D, Pan J, Huang H, Chen G, Qiu S, Shi H, Chen W, Yu Y, Feng S, Chen R. Label-free blood plasma test based on surface-enhanced Raman scattering for tumor stages detection in nasopharyngeal cancer. Scientific Reports 2016; 11(4): 2590-2594.

[11] Artemyev DN, Bratchenko IA, Khristoforova YuA, Lykina AA, Myakinin OO, Kuzmina TP, Davydkin IL, Zakharov VP. Blood proteins analysis by Raman spectroscopy method. Proc. SPIE 9887. Biophotonics: Photonic Solutions for Better Health Care V, 2016.

[12] Boyd S, Bertino MF, Seashols SJ. Raman spectroscopy of blood samples for forensic applications. Forensic Science International 2011; 208(1-3): 124-128.

[13] Shao J, Lin M, Li Y, Li X, Liu J, Liang J, Yao H. In Vivo Blood Glucose Quantification Using Raman Spectroscopy. PLoS One 2012; 7(10).

[14] Pavlova N, Thompson CB. The Emerging Hallmarks of Cancer Metabolism. Cell Metabolism 2016; 23(1): 27-47.

[15] Ashok PC, Singh GP, Tan KM, Dholakia K. Fiber probe based microfluidic Raman spectroscopy. J. Opt Express 2010; 18(8): 7642-7649. DOI: 10.1364/OE.18.007642.

[16] Feng S, Zheng Z, Xu Y, Lin J, Chen G, Weng C, Lin D, Qiu S, Cheng M, Huang Z, Wang L, Chen R, Xie S. A Noninvasive Cancer Detection Strategy Based on Gold Nanoparticle Surface-enhanced Raman Spectroscopy of Urinary Modified Nucleosides Isolated by Affinity Chromatography. Biosensors \& Bioelectronics 2017; 91: 616-622. 